\title{
Spironolacton die beste Option bei "resistenter" Hypertonie
}

\author{
Welche medikamentöse Option verspricht bei „resistenter" Hypertonie den besten Erfolg? \\ Ergebnisse einer neuen Studie sprechen für Spironolacton: Bei fast $60 \%$ der Patienten konnte \\ damit der erhöhte Blutdruck doch noch unter Kontrolle gebracht werden.
}

\begin{abstract}
_ Unter „resistenter“ Hypertonie wird eine Blutdruckerhöhung verstanden, die trotz kombinierter Therapie mit drei Blutdrucksenkern (i.d.R. ACE-Hemmer/Angiotensin-Rezeptor-Antagonist, Kalziumantagonist und Diuretikum) nicht in den Griff zu bekommen ist. Rund $10 \%$ aller Hypertoniker fallen unter diese Kategorie, sie unterliegen einem hohen kardiovaskulären Risiko.

\section{Vergleich mit Alphablocker, Betablocker und Placebo}

Eine Forschergruppe um Prof. Bryan Williams vom University College in London hat nun untersucht, mit welchem vierten Antihypertensivum die besten Chancen bestehen, den Blutdruck doch noch in den Normbereich zu senken. Ihre Hoffnung richtete sich dabei auf den diuretisch wirksamen Aldosteronblocker Spironolacton.
\end{abstract}

Für die PATHWAY-2-Studie wurden 335 Patienten mit resistenter Hypertonie rekrutiert. In vier jeweils zwölfwöchigen Behandlungszyklen wurden sie nacheinander mit Spironolacton, dem Alphablocker Doxazosin, dem Betablocker Bisoprolol und Placebo behandelt. Basis für den Vergleich der Wirksamkeit waren die bei der häuslichen Selbstmessung festgestellten systolischen Blutdruckwerte.

Zunächst erwies sich Spironolacton grundsätzlich als wirksam: Der systolische Blutdruck nahm im Mittel 8,7 mmHg stärker ab als unter Placebo.

Auch den Vergleich mit den aktiven Substanzen gewann der Aldosteronblocker, die Blutdrucksenkung fiel etwa doppelt so deutlich aus. Die Differenzen betrugen $-4,03 \mathrm{mmHg}$ vs. Doxazosin und $-4,48 \mathrm{mmHg}$ vs. Bisoprolol.

Diese Überlegenheit spiegelte sich auch im Anteil der Hypertoniker wider, deren systolische Blutdruckwerte am Ende im Zielbereich unter $135 \mathrm{mmHg}$ lagen. Unter Spironolacton betrug die Rate $57,8 \%$, im Vergleich zu 41,7\% unter Doxazosin, $43,6 \%$ unter Bisoprolol und 24,4\% unter Placebo.

Spironolacton erwies sich als gut verträglich, unerwünschte Effekte traten nicht signifikant häufiger auf als in den Vergleichsgruppen. Bedingung dafür sei allerdings eine sorgfältige Überwachung von Kaliumwerten und Nierenfunktion, betonte Williams.

Peter Overbeck

- HotlineIV beim ESC-Kongress, London, 29.8.-2. 9. 2015

\section{Abklärung von Brustschmerzen}

\section{Neuer Algorithmus bei Infarktverdacht}

_ Ein neuer Algorithmus für die Troponinbestimmung kann die Notfallversorgung bei Herzinfarktverdacht erheblich beschleunigen.

Wenn Patienten mit Brustschmerz in die Notambulanz kommen, wird bisher der kardiale Biomarker Troponin sofort sowie nach drei Stunden gemessen. Bei etwa $40 \%$ der Patienten gibt der Test Entwarnung. Bei gut 10\% spricht der Test für ein akutes Koronargeschehen (ACS), sie werden entsprechend versorgt. Alle übrigen Patienten müssen stationär weiter abgeklärt werden.
PD Dr. Dirk Westermann, Universitätsklinikum Hamburg Eppendorf, präsentierte die Ergebnisse der BACC (Biomarkers in Acute Cardiovasculare Care)-Studie. In dieser wurde der bisherige 3-Stunden-Algorithmus mit einem neuen 1-Stunden-Algorithmus verglichen, bei dem das hochsensitive Troponin I bestimmt wird. Für Patienten mit negativem Test kann deutlich früher Entwarnung gegeben werden.

Beide Tests erwiesen sich als gleichwertig, so Westermann. Der Ein-Stunden-Algorithmus gab bei $40 \%$ der Patienten Ent- warnung, negativer prädiktiver Wert und Sensitivität lagen jeweils bei über $99 \%$. Als Troponin-I-Grenzwert wurde 6 ng/l gewählt. Das Vorgehen wurde an weiteren Kohorten validiert, der zuverlässige ACSAusschluss bestätigte sich. Somit kann das Verfahren zur Ausschlussdiagnostik eingesetzt werden, so Westermann. Der positiv-prädiktive Wert wurde mit $87 \%$ bei einer Spezifität von $98 \%$ berechnet. Auch dies sei nicht schlechter als beim bisherigen dreistündigen Vorgehen.

Philipp Grätzel

- Hotline I, ESC-Kongress London 29.8.-2.9.2015 